Supporting Information

\title{
From Planar Macrocycle to Cylindrical Molecule: Synthesis and Properties of A Phenanthrene-Based Coronal Nanohoop as A Segment of $[6,6]$ Carbon Nanotube
}

Shengsheng Cui, Qiang Huang, Jinyi Wang, Hongxing Jia, Pingsen Huang, Shengda

Wang, Pingwu $\mathrm{Du}^{*}$

Hefei National Laboratory for Physical Sciences at the Microscale, CAS Key Laboratory of Materials for Energy Conversion, $i \mathrm{ChEM}$, Department of Materials Science and Engineering, University of Science and Technology of China (USTC), 96 Jinzhai Road, Hefei, Anhui Province, 230026, China

*To whom correspondence should be addressed

E-mail: dupingwu@ustc.edu.cn

Tel/Fax: 86-551-63606207 


\section{Materials and General Information}

All solvents for syntheses were obtained from commercial suppliers (Innochem or Acros) and used without further purification. Air-sensitive reactions were all carried out under argon. Flash column chromatography was performed with 200-300 mesh silica gel using eluents as specified. NMR spectra were recorded on Bruker BioSpin $\left({ }^{1} \mathrm{H} 400 \mathrm{MHz},{ }^{13} \mathrm{C} 100 \mathrm{MHz}\right)$ spectrometer, and chemical shifts were reported as the delta scale in ppm relative to $\mathrm{CDCl}_{3}(\delta=7.26 \mathrm{ppm})$ for ${ }^{1} \mathrm{H} \mathrm{NMR}$ and $\mathrm{CDCl}_{3}(\delta=77.16$ ppm) for ${ }^{13} \mathrm{C}$ NMR. Data are reported as follows: chemical shift, multiplicity ( $\mathrm{s}=$ singlet, $\mathrm{d}=$ doublet, $\mathrm{t}=$ triplet, $\mathrm{m}=$ multiplet, $\mathrm{br}=$ broad signal), coupling constant $(\mathrm{Hz})$, and integration. High resolution mass spectrometry (HR-MS) analyses were carried out using MALDI-TOF-MS techniques (matrix, DCTB). Preparative thin-layer chromatography (PTLC) experiments were performed using silica gel GF 254 coated plates. UV-Vis absorption spectra were performed on a UNIC-3802 spectrophotometer. 


\section{Synthesis procedures}

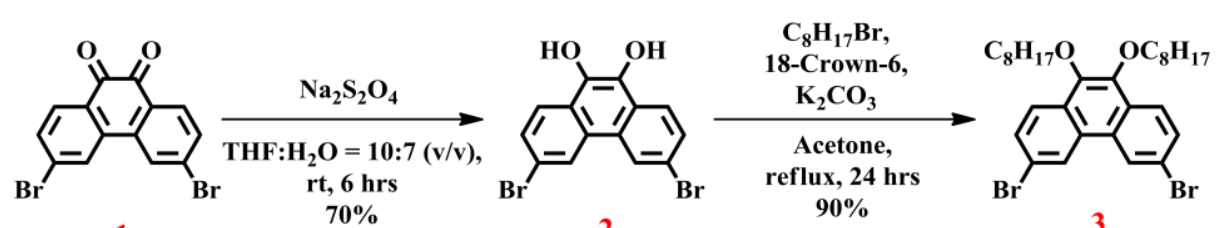

1

2

3
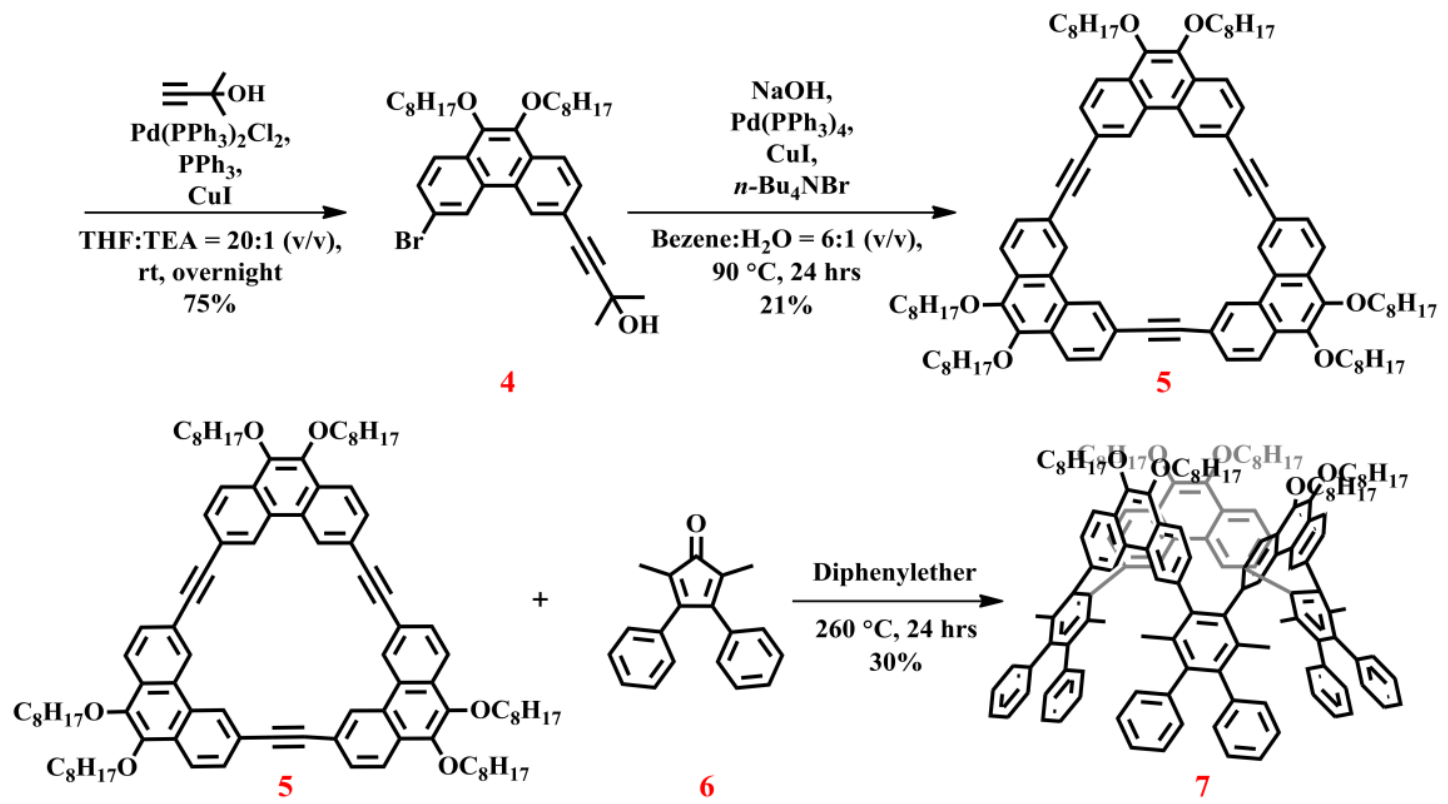

\section{Synthesis of 3,6-dibromophenanthrene-9,10-diol (2) ${ }^{\mathrm{S} 1}$}

3,6-dibromophenanthrene-9,10-dione 1 (2.0 g, $5.5 \mathrm{mmol})$ and $\mathrm{Na}_{2} \mathrm{~S}_{2} \mathrm{O}_{4}(3.74 \mathrm{~g}, 21.5$ mmol) were dissolved into a mixture of THF $(100 \mathrm{~mL})$ and $\mathrm{H}_{2} \mathrm{O}(70 \mathrm{~mL})$. The mixture stirred at room temperature for $6 \mathrm{~h}$. After removing the solvent, the crude solid (1.4 g, $70 \%$ ) was collected by filtration, washed with water and dried in vacuum for the next step without further purification.

\section{Synthesis of 3,6-dibromo-9,10-bis(octyloxy)phenanthrene $(3)^{\mathrm{S} 2}$}

To a mixture of 3, 6-dibromophenanthrene-9, 10-diol 2 (2.0 g, 5.4mmol), $\mathrm{K}_{2} \mathrm{CO}_{3}(2.3$ $\mathrm{g}, 16.6 \mathrm{mmol})$, and 18 -crown-6 (200 $\mathrm{mg}, 0.76 \mathrm{mmol})$ in $40 \mathrm{~mL}$ acetone, which was purged with argon for 20 minutes, was added 1-bromooctane (2.66 g, $13.8 \mathrm{mmol})$ via a syringe under an argon atmosphere. The resulting solution was heated to reflux 
temperature for $24 \mathrm{~h}$ under argon atmosphere. The crude product was purified by column chromatography (silica gel, pure petroleum ether (PE)) to afford $\mathbf{3}$ as a white solid (2.9 g, 90\%). ${ }^{1} \mathrm{H}$ NMR (400 MHz, $\left.\mathrm{CDCl}_{3}\right): \delta 8.64(\mathrm{~d}, J=1.9 \mathrm{~Hz}, 2 \mathrm{H}), 8.09$ (d, $J$ $=8.7 \mathrm{~Hz}, 2 \mathrm{H}), 7.70\left(\mathrm{dd}, J_{1}=8.8 \mathrm{~Hz}, J_{2}=1.9 \mathrm{~Hz}, 2 \mathrm{H}\right), 4.17(\mathrm{t}, J=6.7 \mathrm{~Hz}, 4 \mathrm{H}), 1.92-$ $1.84(\mathrm{~m}, 4 \mathrm{H}), 1.40-1.27(\mathrm{~m}, 20 \mathrm{H}), 0.90(\mathrm{t}, J=6.9 \mathrm{~Hz}, 6 \mathrm{H})$.

Synthesis of 4-(6-bromo-9,10-bis(octyloxy)phenanthren-3-yl)-2-methylbut-3-yn2-ol (4) $)^{\mathrm{S} 3-\mathrm{S} 5}$

To a mixture of 3,6-dibromo-9,10-bis(octyloxy)phenanthrene 3 (2.7 g, $4.56 \mathrm{mmol}$ ), CuI (180.0 mg, $0.95 \mathrm{mmol}), \mathrm{PPh}_{3}(250 \mathrm{mg}, 0.95 \mathrm{mmol}), \mathrm{Pd}\left(\mathrm{PPh}_{3}\right)_{2} \mathrm{Cl}_{2}(100 \mathrm{mg}, 0.14 \mathrm{mmol})$ and $3 \mathrm{~mL}$ of triethylamine in $60 \mathrm{~mL}$ of oxygen-free THF was added 2-methylbut-3-yn2-ol (400 mg, $4.75 \mathrm{mmol})$ via a syringe under an argon atmosphere at room temperature. The resulting solution was stirred at room temperature overnight under an argon atmosphere. The reaction mixture was then extracted with diethyl ether for three times. The organic layers were combined, washed with water and brine, dried with anhydrous $\mathrm{Na}_{2} \mathrm{SO}_{4}$ and concentrated under reduced pressure. The crude product was purified by column chromatography (silica gel, $\left.\mathrm{CH}_{2} \mathrm{Cl}_{2}\right)$ to afford 4 as a light yellow oil (2.0 g, 75\%). ${ }^{1} \mathrm{H}$ NMR (400 MHz, $\left.\mathrm{CDCl}_{3}\right): \delta 8.71(\mathrm{~d}, J=1.9 \mathrm{~Hz}, 1 \mathrm{H}), 8.59(\mathrm{~d}, J=1.4 \mathrm{~Hz}, 1 \mathrm{H})$, $8.15(\mathrm{~d}, J=8.5 \mathrm{~Hz}, 1 \mathrm{H}), 8.09(\mathrm{~d}, J=8.7 \mathrm{~Hz}, 1 \mathrm{H}), 7.69\left(\mathrm{dd}, J_{1}=8.7 \mathrm{~Hz}, J_{2}=1.8 \mathrm{~Hz}\right.$, 1H), $7.62\left(\mathrm{dd}, J_{1}=8.5 \mathrm{~Hz}, J_{2}=1.5 \mathrm{~Hz}, 1 \mathrm{H}\right), 4.20-4.15(\mathrm{~m}, 4 \mathrm{H}), 2.09(\mathrm{~s}, 1 \mathrm{H}), 1.92-1.83$ (m, 4H), $1.70(\mathrm{~s}, 6 \mathrm{H}), 1.54-1.22(\mathrm{~m}, 20 \mathrm{H}), 0.90(\mathrm{t}, J=6.9 \mathrm{~Hz}, 6 \mathrm{H})$.

\section{Synthesis of compound 5}

To a mixture of 4-(6-bromo-9,10-bis(octyloxy)phenanthren-3-yl)-2-methylbut-3-yn-2- 
ol 4 (800 mg, $1.34 \mathrm{mmol})$, CuI (12.8 mg, $0.067 \mathrm{mmol}), n-\mathrm{Bu} 4 \mathrm{NBr}(21.6 \mathrm{mg}, 0.067$ $\mathrm{mmol})$ and $\mathrm{Pd}\left(\mathrm{PPh}_{3}\right)_{4}(77.4 \mathrm{mg}, 0.067 \mathrm{mmol})$ were added $30 \mathrm{~mL}$ of benzene and $5 \mathrm{~mL}$ of $5 \mathrm{M} \mathrm{NaOH}$ aqueous solution, both of which were purged with argon before use. The reaction mixture was heated at $90{ }^{\circ} \mathrm{C}$ under an argon atmosphere for 24 hours, and then cooled to room temperature. The crude product was then extracted with diethyl ether for three times. The organic layers were combined, washed with water and brine, dried with anhydrous $\mathrm{Na}_{2} \mathrm{SO}_{4}$ and concentrated under reduced pressure. The crude product was purified by column chromatography (silica gel, $\mathrm{PE} / \mathrm{CH}_{2} \mathrm{Cl}_{2}=1 / 3$ ) to afford 5 as a yellow solid (130 mg, 21\%). Mp: 49-50 ㄷ. IR (KBr): 2954, 2927, 2854, 1603, 1552 , $1496,1467,1427,1382,1354,1319,1285,1269,1236,1173,1121,1054,877,825$, $722 \mathrm{~cm}^{-1} .{ }^{1} \mathrm{H}$ NMR $\left(400 \mathrm{MHz}, \mathrm{CDCl}_{3}\right): \delta 9.26(\mathrm{~s}, 6 \mathrm{H}), 8.21(\mathrm{~d}, J=8.4 \mathrm{~Hz}, 6 \mathrm{H}), 7.79$ $\left(\mathrm{dd}, J_{1}=8.5 \mathrm{~Hz}, J_{2}=1.2 \mathrm{~Hz}, 6 \mathrm{H}\right), 4.25-4.20(\mathrm{~m}, 12 \mathrm{H}), 1.97-1.89(\mathrm{~m}, 12 \mathrm{H}), 1.58-1.62$ (m, 6H), 1.50-1.25 (m, 54H), $0.92(\mathrm{t}, J=6.9 \mathrm{~Hz}, 18 \mathrm{H}) .{ }^{13} \mathrm{C} \mathrm{NMR}\left(100 \mathrm{MHz}, \mathrm{CDCl}_{3}\right)$ : $\delta 14.30,22.87,26.46,29.54,29.73,30.70,32.06,73.85,91.17,120.87,122.51,127.13$, $128.13,129.43,129.50,144.07$. HR-MS (MALDI-TOF-MS) $m / z$ calcd. for $\mathrm{C}_{96} \mathrm{H}_{120} \mathrm{O}_{6}$ $[\mathrm{M}]^{+}:$1369.9118, Found 1369.8900. UV-Vis: $\lambda_{\max }: 334 \mathrm{~nm}, 351 \mathrm{~nm}, 386 \mathrm{~nm}, 407 \mathrm{~nm}$.

\section{Synthesis of compound 7}

A 150-mL flask containing compound 5 (100 mg, $0.073 \mathrm{mmol})$ and 2,5-dimethyl-3,4diphenylcyclopenta-2,4-dienone 6 (115 mg, $0.44 \mathrm{mmol}$ ) which was synthesized following the reported procedure ${ }^{\mathrm{S} 6}$ was evacuated and refilled with argon for 3 cycles, and then diphenylether $(2 \mathrm{~mL})$ was transferred to the flask via syringe under argon at room temperature. The mixture was then stirred at $260{ }^{\circ} \mathrm{C}$ for $24 \mathrm{~h}$. After cooling down 
to room temperature, methanol $(100 \mathrm{~mL})$ was added to obtain the precipitate. The residue was purified initially by silica gel column chromatography and then by preparative thin-layer chromatography $\left(\mathrm{PE} / \mathrm{CH}_{2} \mathrm{Cl}_{2}=1 / 4, v / v\right)$ to afford the final product 7 as a pale yellow solid (45 mg, 30\%). Mp: $182-184{ }^{\circ} \mathrm{C}$. IR (KBr): 2954, 2925, $2854,1604,1508,1457,1441,1397,1379,1353,1323,1314,1234,1173,1121,1051$, 889, 829, 773, 742, $700 \mathrm{~cm}^{-1} .{ }^{1} \mathrm{H}$ NMR (400 MHz, $\left.\mathrm{CDCl}_{3}\right): \delta 8.27(\mathrm{~s}, 6 \mathrm{H}), 7.99(\mathrm{~d}, J=$ $8.3 \mathrm{~Hz}, 6 \mathrm{H}), 7.61(\mathrm{~d}, J=8.3 \mathrm{~Hz}, 6 \mathrm{H}), 7.24-7.19(\mathrm{~m}, 6 \mathrm{H}), 7.14-7.06(\mathrm{~m}, 24 \mathrm{H}), 4.06-3.95$ (m, 12H), 1.87-1.80 (m, 12H), $1.69(\mathrm{~s}, 18 \mathrm{H}), 1.52-1.47(\mathrm{~m}, 6 \mathrm{H}), 1.38-1.26(\mathrm{~m}, 54 \mathrm{H})$, $0.90(\mathrm{t}, J=6.0 \mathrm{~Hz}, 18 \mathrm{H}) .{ }^{13} \mathrm{C} \mathrm{NMR}\left(100 \mathrm{MHz}, \mathrm{CDCl}_{3}\right): \delta 14.28,19.87,22.84,26.38$, $29.50,29.68,30.67,32.02,73.64,121.22,123.94,126.02,127.59,127.63,127.96$, $128.39,129.90,130.31,130.45,132.20,138.52,141.01,141.27,141.79,143.00$. HRMS (MALDI-TOF-MS) m/z calcd. for $\mathrm{C}_{150} \mathrm{H}_{168} \mathrm{O}_{6}[\mathrm{M}]^{+}:$2066.2874, Found 2066.2906. UV-Vis: $\lambda_{\max }: 306 \mathrm{~nm}, 318 \mathrm{~nm}, 350 \mathrm{~nm}, 367 \mathrm{~nm}$.

Computational detail. All DFT calculations were performed using the $\mathrm{DMol}^{3}$ module in the Material Studio program. ${ }^{\text {S7-S8 }}$ Exchange-correlation effect term in Kohn-shame equation were treated by using gradient-corrected BLYP (Becke exchange plus LeeYang-Parr correlation) functional. ${ }^{\mathrm{S} 9}$ All electrons numerical basis sets of DNP (Double Numerical plus polarization), ${ }^{\mathrm{S} 10}$ featuring the more accuracy than Gaussian $6-31 \mathrm{G}^{* *}$ basis set, was used to describe the atomic orbitals of $\mathrm{C}, \mathrm{H}$ and $\mathrm{O}$ atoms. Moreover, dispersion energy was corrected by Grimme method. ${ }^{\text {S11 }}$ For the optimization calculations, the convergence in energy and force was set to be $1.0 \times 10^{-5} \mathrm{Ha}$ and $1.0 \times$ $10^{-3} \mathrm{Ha} . \AA^{-1}$, and the SCF convergence was set to be $1.0 \times 10^{-6}$. The tension energy 
was calculated based on the reported computational method. ${ }^{\mathrm{S} 12}$ To reduce computational cost, six $\mathrm{C}_{8} \mathrm{H}_{17}$ functional groups in phenanthrene-based coronal nanohoop 7 was replaced by methyl groups.

Fluorescence quenching experiment. To a solution of phenanthrene-based coronal nanohoop $7\left(1.0 \times 10^{-5} \mathrm{~mol} \mathrm{~L}^{-1}\right)$ were titrated by $\mathrm{C}_{60}\left(5 \times 10^{-5} \mathrm{~mol} \mathrm{~L}^{-1}\right)$ in toluene at room temperature. The changes in the fluorescent intensity of phenanthrene-based coronal nanohoop 7 at different ratios between them were recorded. The $K_{\mathrm{a}}$ was determined by the equation: ${ }^{\mathrm{S} 13-\mathrm{S} 15} F / F_{0}=\left(1+\left(k_{\mathrm{f}} / k_{\mathrm{s}}\right) K_{a}\left[\mathrm{C}_{60}\right]\right) /\left(1+K_{a}\left[\mathrm{C}_{60}\right]\right) . F, F_{0}, k_{\mathrm{f}}, k_{\mathrm{s}}$, $K_{a},\left[\mathrm{C}_{60}\right]$ are fluorescence intensity, fluorescence of coronal nanohoop 7 before the addition of $\mathrm{C}_{60}$, a proportionality constant of the complex, a proportionality constant of the host, the binding constant, and the concentration of $\mathrm{C}_{60}$, respectively.

Job's plot. A solution of $\mathrm{C}_{60}$ in toluene and a solution of coronal nanohoop 7 in toluene were mixed in different ratios to prepare 11 samples $\left(\left[\mathrm{C}_{60}\right]+[7]=1.0 \times 10^{-5} \mathrm{~mol} \mathrm{~L}^{-1}\right)$. The UV-Vis absorption spectra were recorded for each sample, and the absorption intensity changes at $336 \mathrm{~nm}$ were monitored for Job's plot analysis. The results are summarized in Figure $6 b$. 


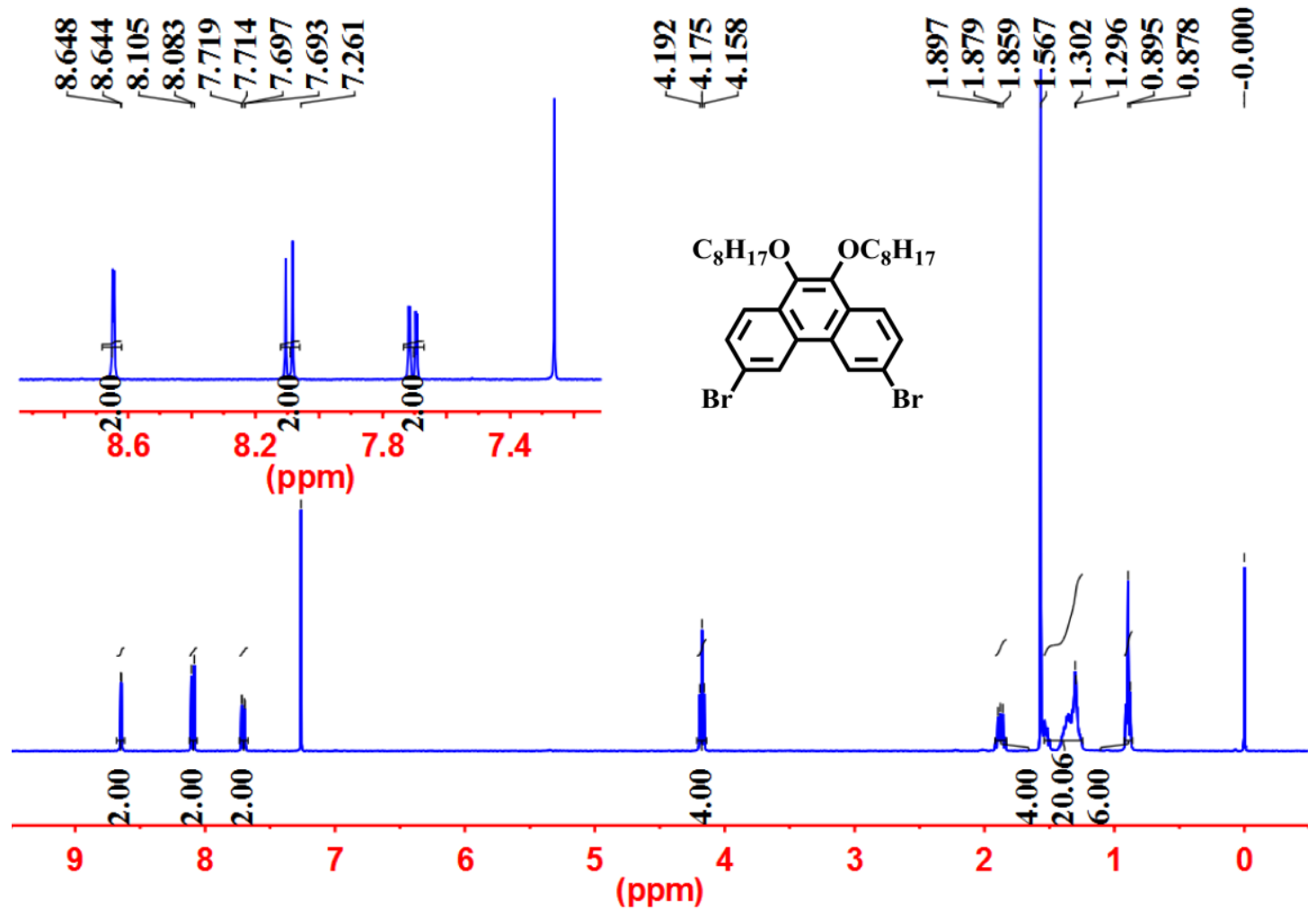

Figure S1. ${ }^{1} \mathrm{H}$ NMR spectrum of 3,6-dibromo-9,10-bis(octyloxy)phenanthrene 3 in $\mathrm{CDCl}_{3}$. 


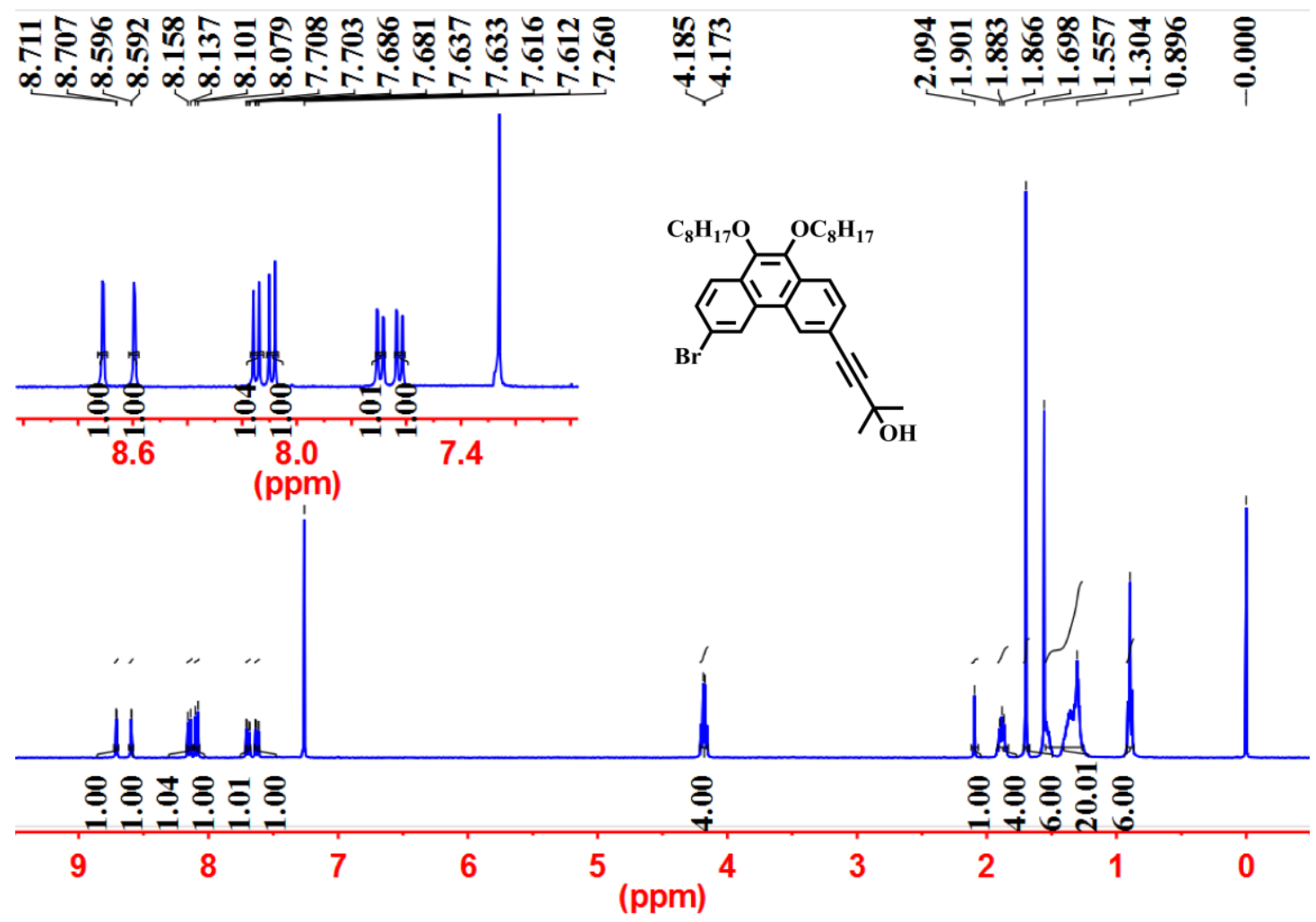

Figure S2. ${ }^{1} \mathrm{H}$ NMR spectrum of 4-(6-bromo-9, 10-bis(octyloxy)phenanthren-3yl)-2-methylbut-3-yn-2-ol 4 in $\mathrm{CDCl}_{3}$. 


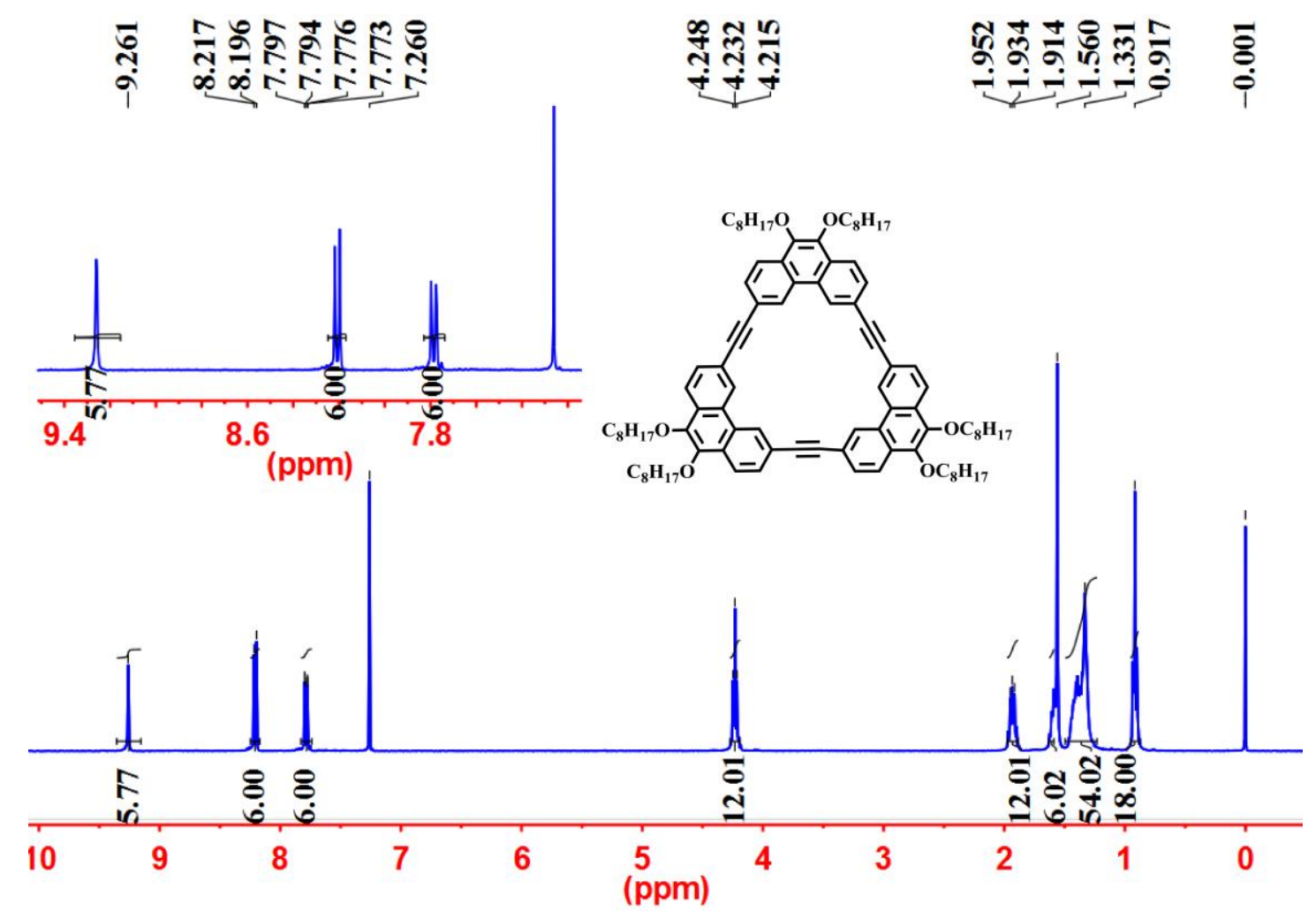

Figure S3. ${ }^{1} \mathrm{H}$ NMR spectrum of aryleneethynylene macrocycle 5 in $\mathrm{CDCl}_{3}$. 


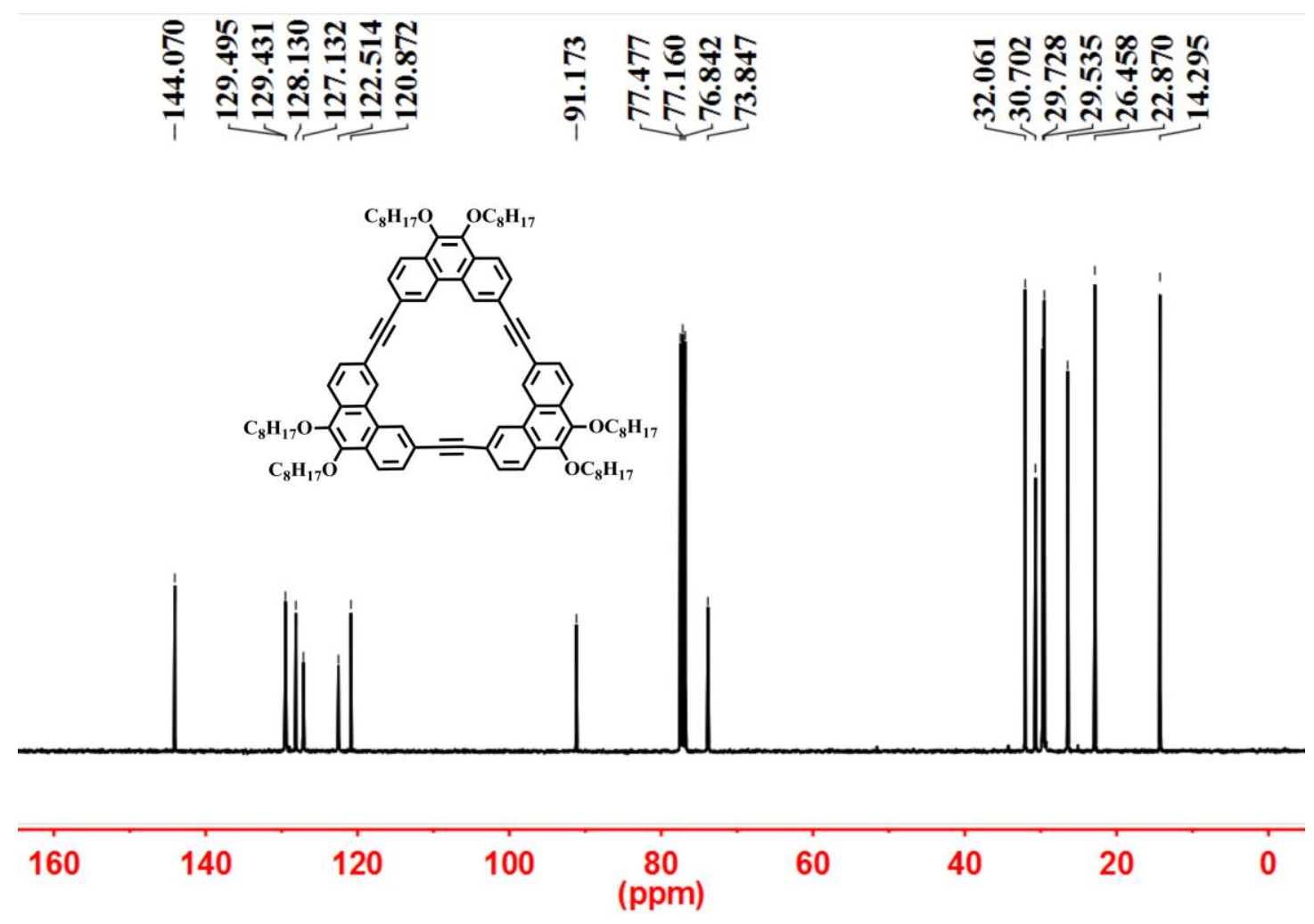

Figure S4. ${ }^{13} \mathrm{C}$ NMR spectrum of aryleneethynylene macrocycle 5 in $\mathrm{CDCl}_{3}$ 


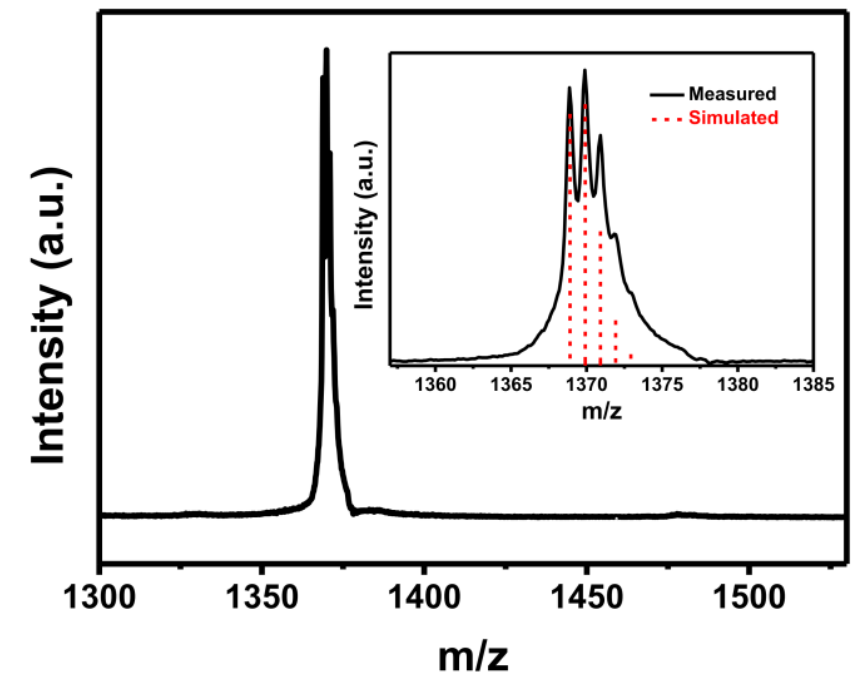

Figure S5. MALDI-TOF-MS and simulated data for aryleneethynylene macrocycle 5. 


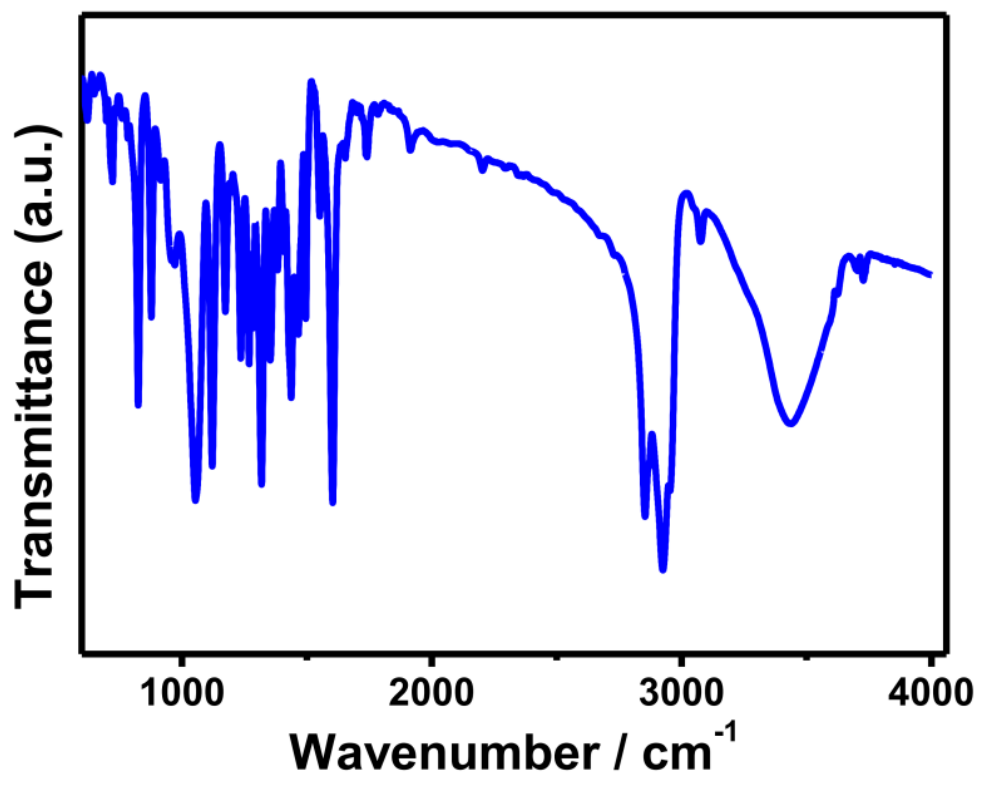

Figure S6. FTIR spectrum of aryleneethynylene macrocycle 5. 


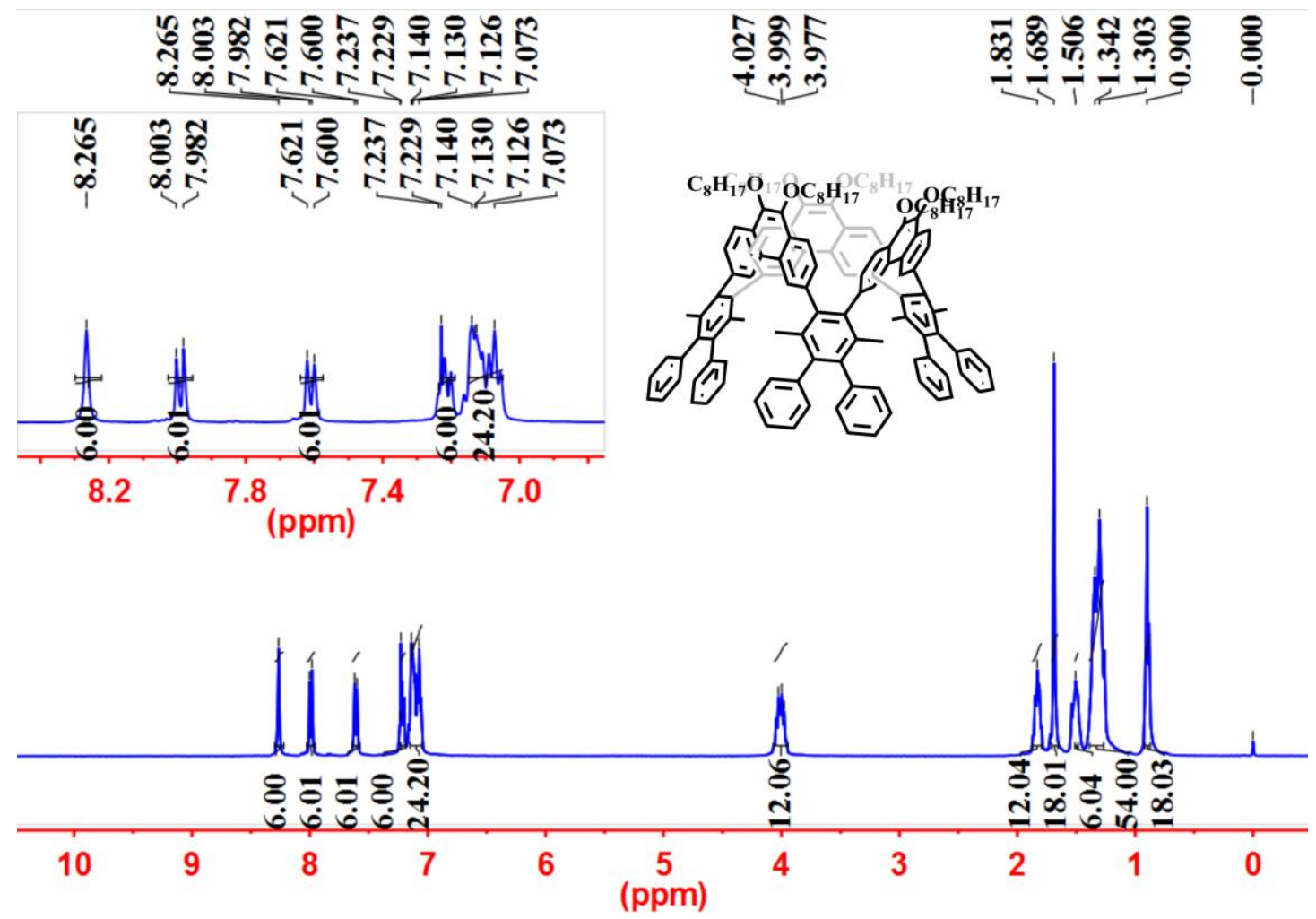

Figure S7. ${ }^{1} \mathrm{H}$ NMR spectrum of phenanthrene-based coronal nanohoop 7 in $\mathrm{CDCl}_{3}$ 


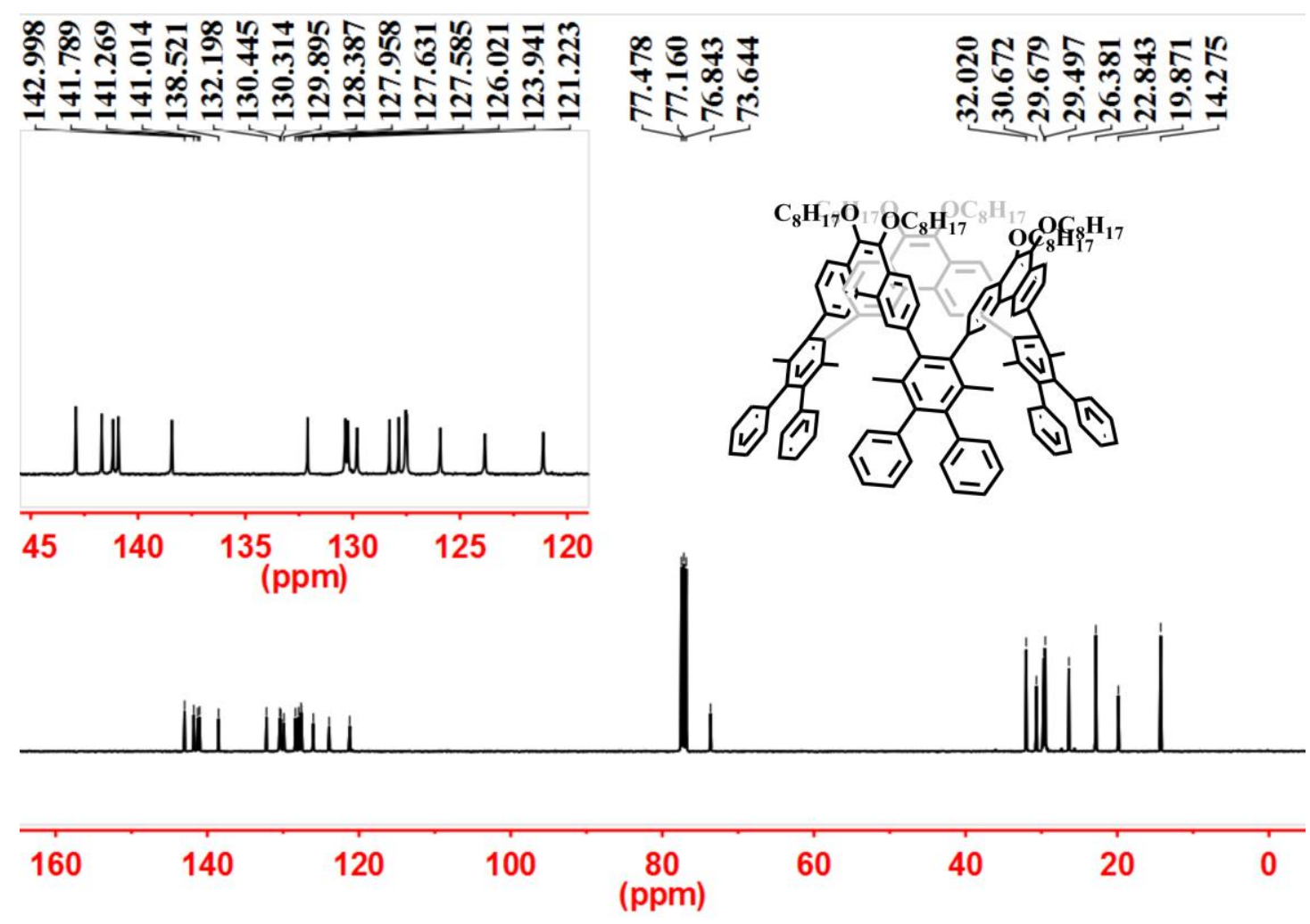

Figure S8. ${ }^{13} \mathrm{C}$ NMR spectrum of coronal nanohoop 7 in $\mathrm{CDCl}_{3}$. 


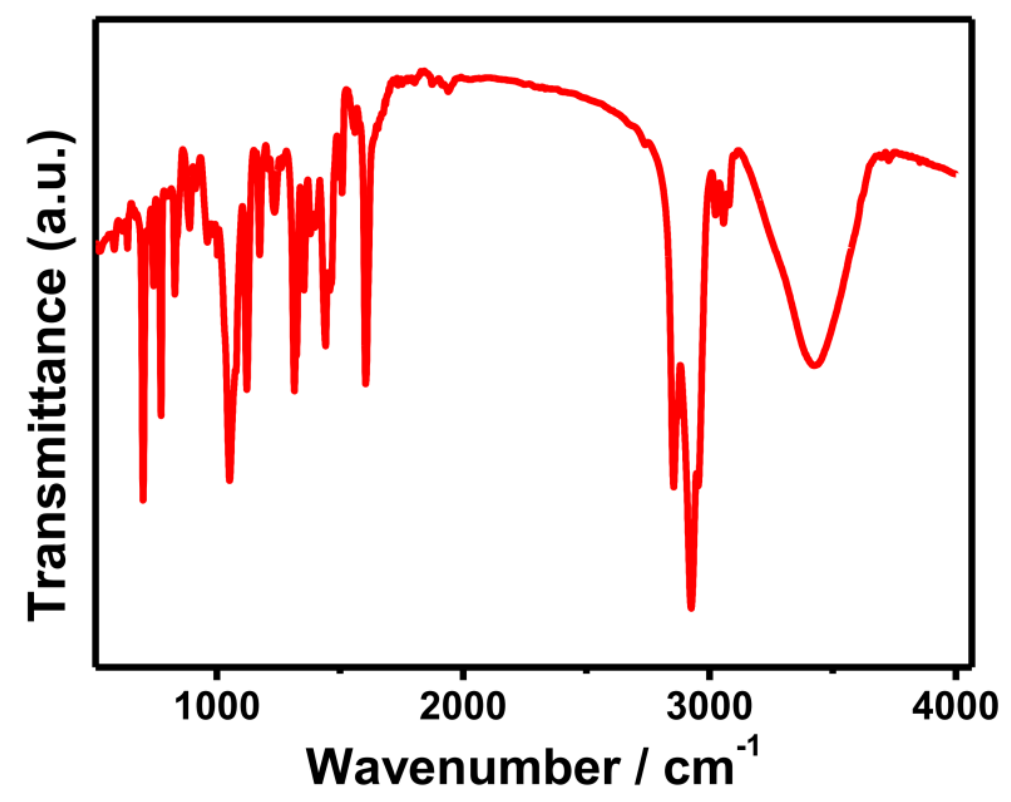

Figure S9. FTIR spectrum of coronal nanohoop 7. 


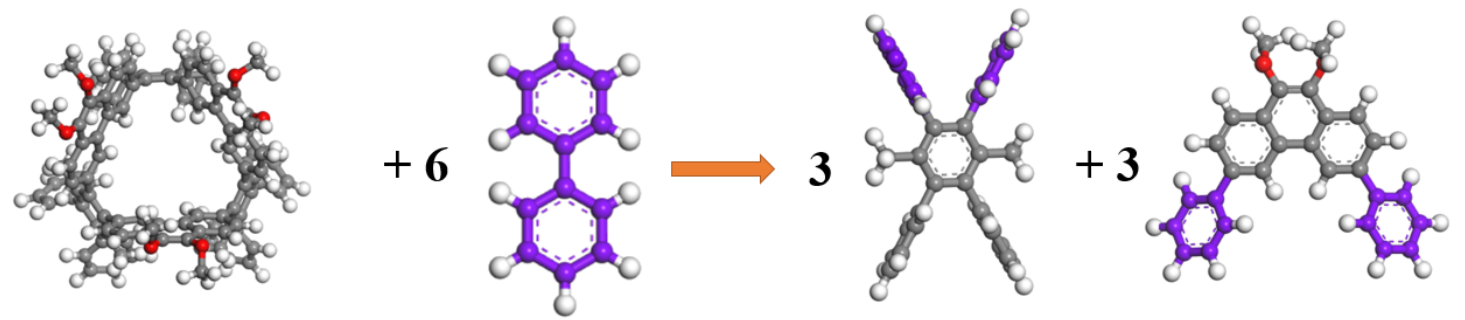

Figure S10. Homodesmotic reactions used for strain energy calculation. 


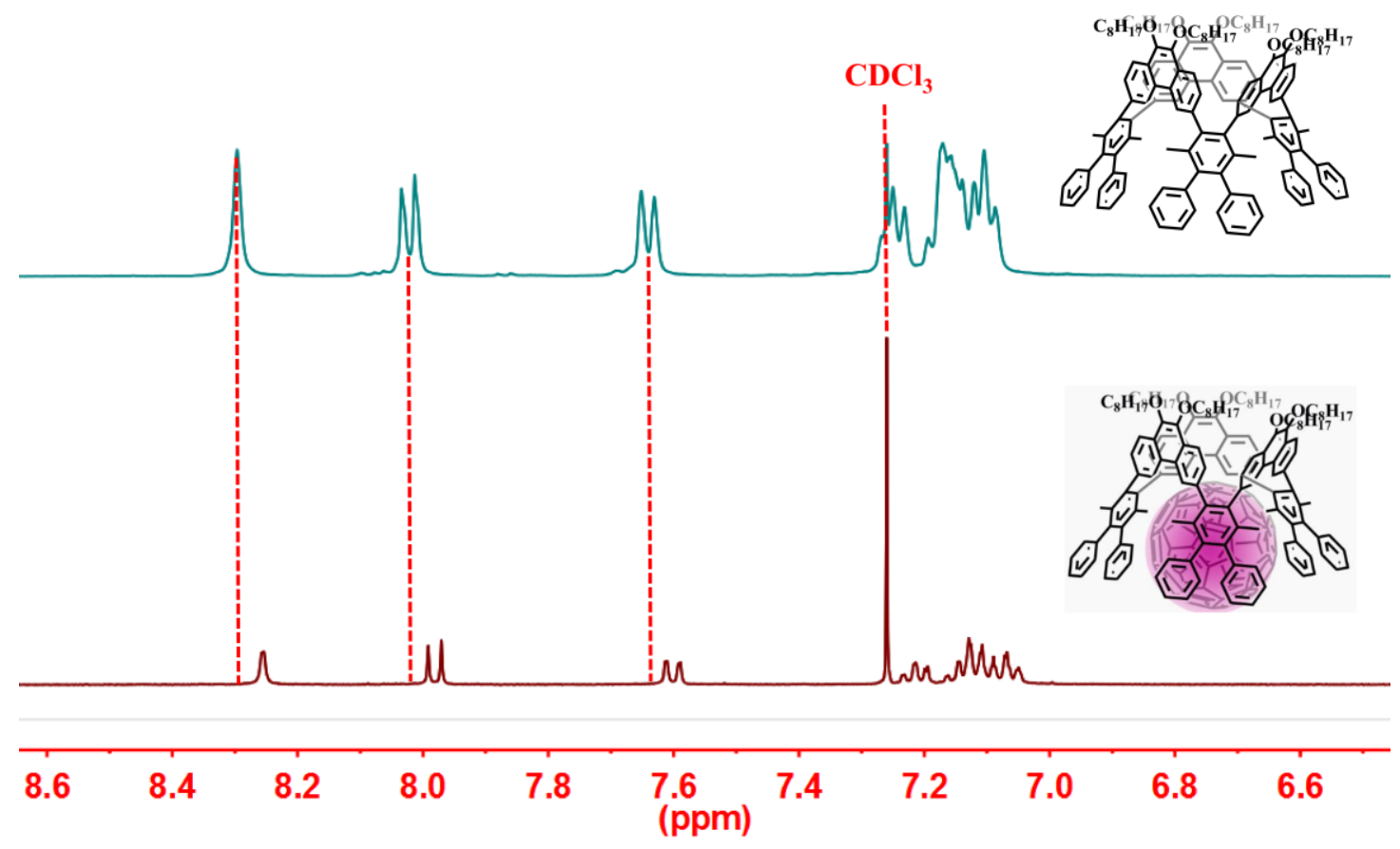

Figure S11. ${ }^{1} \mathrm{H}$ NMR spectra of coronal nanohoop 7 in $\mathrm{CDCl}_{3}$ at room temperature before (a) and after (b) the addition of $\mathrm{C}_{60}$. 
Table S1. Calculated Energy data of different moieties (unit: Hatree) and strain energies (unit: $\mathrm{kcal} / \mathrm{mol}$ )

\begin{tabular}{cc}
\hline Molecules & Energy/ Hartree \\
\hline Coronal nanohoop 7 & -4617.581451 \\
Biphenyl & -463.2877507 \\
1,4-dimethylbenzene moiety & -1235.056075 \\
Phenanthrene moiety & -1230.714674 \\
Strain energy( kcal/mol ) & 2.69 \\
\hline
\end{tabular}


Table S2. Coordinate of relaxed coronal nanohoop 7

\begin{tabular}{|c|c|c|c|c|c|c|c|}
\hline \multicolumn{8}{|c|}{ Relaxed structure of coronal nanohoop 7} \\
\hline $\mathbf{C}$ & 6.861945 & -4.512504 & -0.538725 & $\mathrm{C}$ & -6.561345 & 1.037274 & 1.941931 \\
\hline $\mathbf{C}$ & 1.218692 & -8.148236 & -0.676706 & $\mathrm{C}$ & -0.220078 & 6.713977 & 1.430572 \\
\hline $\mathbf{C}$ & -1.779372 & -6.592581 & -4.357070 & $\mathrm{C}$ & 4.831598 & 2.844462 & -0.604761 \\
\hline $\mathbf{C}$ & -1.385598 & -1.042420 & -8.059744 & $\mathrm{O}$ & -7.369107 & 3.201356 & 1.245648 \\
\hline $\mathbf{C}$ & 3.096498 & 0.943220 & -7.328721 & $\mathrm{C}$ & -8.397892 & 3.276665 & 0.220139 \\
\hline C & 7.800439 & -0.262353 & -2.910599 & $\mathrm{O}$ & -7.461552 & 0.899901 & 2.981120 \\
\hline C & 5.682469 & -3.997843 & -1.095530 & $\mathrm{C}$ & -8.853406 & 1.243701 & 2.764170 \\
\hline C & 2.508282 & -7.606947 & -0.778587 & $\mathrm{O}$ & 1.087286 & 7.253317 & 3.393195 \\
\hline C & -0.927724 & -5.533727 & -4.011800 & $\mathrm{C}$ & 0.047714 & 7.288819 & 4.414949 \\
\hline C & -1.497797 & -2.342710 & -7.547069 & $\mathrm{O}$ & 0.578145 & 1.260301 & 7.957812 \\
\hline C & 2.763197 & 0.131150 & -6.235256 & $\mathrm{C}$ & 0.884351 & 0.503208 & 9.164539 \\
\hline C & 7.001031 & -0.046681 & -4.042397 & $\mathrm{O}$ & -1.004732 & 7.855895 & 1.565497 \\
\hline C & 2.720346 & -6.256937 & -0.464376 & $\mathrm{C}$ & -0.275924 & 9.105111 & 1.389478 \\
\hline C & -0.970345 & -4.989689 & -2.721841 & $\mathrm{O}$ & -1.727242 & -0.380777 & 7.838074 \\
\hline C & -1.591391 & -2.536575 & -6.161310 & $\mathrm{C}$ & -2.503348 & 0.841030 & 8.010481 \\
\hline C & 1.662636 & 0.453087 & -5.430640 & $\mathrm{H}$ & 7.260988 & -5.465629 & -0.883598 \\
\hline C & 5.655737 & 0.314088 & -3.882448 & $\mathrm{H}$ & 1.044618 & -9.195982 & -0.920563 \\
\hline C & 5.174373 & -2.769202 & -0.654095 & $\mathrm{H}$ & -1.745401 & -7.021040 & -5.357958 \\
\hline C & 7.529589 & -3.788757 & 0.459962 & $\mathrm{H}$ & -1.313984 & -0.882519 & -9.135182 \\
\hline C & 0.147786 & -7.345059 & -0.266270 & $\mathrm{H}$ & 3.951289 & 0.694120 & -7.956325 \\
\hline C & -2.671771 & -7.102865 & -3.402650 & $\mathrm{H}$ & 8.847095 & -0.542409 & -3.026963 \\
\hline C & -1.370102 & 0.057118 & -7.193311 & $\mathrm{H}$ & 5.159640 & -4.549041 & -1.875846 \\
\hline C & 2.318574 & 2.074912 & -7.614062 & $\mathrm{H}$ & 3.340814 & -8.233022 & -1.098469 \\
\hline C & 7.260259 & -0.118159 & -1.626565 & $\mathrm{H}$ & -0.228482 & -5.132504 & -4.743907 \\
\hline C & 7.019275 & -2.559403 & 0.897333 & $\mathrm{H}$ & -1.509742 & -3.197745 & -8.222443 \\
\hline C & 0.347153 & -5.989861 & 0.053686 & $\mathrm{H}$ & 3.360013 & -0.750665 & -6.006952 \\
\hline C & -2.710404 & -6.556852 & -2.113403 & $\mathrm{H}$ & 7.423987 & -0.160111 & -5.040161 \\
\hline C & -1.463405 & -0.123004 & -5.800929 & $\mathrm{H}$ & 3.716632 & -5.822669 & -0.532906 \\
\hline C & 1.217659 & 2.392408 & -6.807821 & $\mathrm{H}$ & -0.300704 & -4.176532 & -2.447539 \\
\hline C & 5.911818 & 0.242627 & -1.451968 & $\mathrm{H}$ & -1.681362 & -3.539867 & -5.747656 \\
\hline C & 5.836588 & -2.035427 & 0.346080 & $\mathrm{H}$ & 1.392840 & -0.184331 & -4.590613 \\
\hline C & 1.646425 & -5.457941 & -0.052810 & $\mathrm{H}$ & 5.020923 & 0.487882 & -4.749954 \\
\hline C & -1.860926 & -5.494309 & -1.757904 & $\mathrm{H}$ & 4.267059 & -2.361188 & -1.095701 \\
\hline C & -1.576014 & -1.433507 & -5.298302 & $\mathrm{H}$ & 8.450336 & -4.178155 & 0.893447 \\
\hline C & 0.876171 & 1.585460 & -5.707948 & $\mathrm{H}$ & -0.853399 & -7.764433 & -0.196152 \\
\hline C & 5.120063 & 0.457339 & -2.596787 & $\mathrm{H}$ & -3.332674 & -7.929633 & -3.661368 \\
\hline C & -1.881958 & -4.896146 & -0.384012 & $\mathrm{H}$ & -1.271842 & 1.064562 & -7.591510 \\
\hline C & -0.302569 & 1.896854 & -4.835520 & $\mathrm{H}$ & 2.567236 & 2.708015 & -8.465256 \\
\hline C & 5.278974 & -0.718325 & 0.793481 & $\mathrm{H}$ & 7.881083 & -0.296025 & -0.751271 \\
\hline C & -0.789989 & -5.119888 & 0.490444 & $\mathrm{H}$ & 7.540361 & -1.992044 & 1.667608 \\
\hline C & -1.433572 & 1.043590 & -4.864117 & $\mathrm{H}$ & -3.398146 & -6.955595 & -1.368588 \\
\hline C & 5.306540 & 0.388211 & -0.090836 & $\mathrm{H}$ & 0.606578 & 3.265742 & -7.032675 \\
\hline C & 4.565739 & -1.822917 & 2.975428 & $\mathrm{H}$ & 1.814124 & -4.410040 & 0.191997 \\
\hline C & -0.754567 & -4.499112 & 1.759978 & $\mathrm{H}$ & -1.643721 & -1.587640 & -4.222422 \\
\hline C & -4.093166 & -3.758247 & -0.956328 & $\mathrm{H}$ & 4.074123 & 0.734441 & -2.474377 \\
\hline C & -2.516730 & 1.271291 & -3.984876 & $\mathrm{H}$ & 5.494755 & -2.401833 & 2.950841 \\
\hline
\end{tabular}




\begin{tabular}{|c|c|c|c|c|c|c|c|}
\hline $\mathbf{C}$ & 0.998825 & 3.858285 & -3.868003 & $\mathrm{H}$ & 3.756944 & -2.503597 & 2.664568 \\
\hline $\mathrm{C}$ & 4.719643 & 1.617449 & 0.290245 & $\mathrm{H}$ & 4.359302 & -1.518497 & 4.007136 \\
\hline $\mathrm{C}$ & 4.639523 & -0.616840 & 2.049719 & $\mathrm{H}$ & -4.303297 & -4.621464 & -1.595439 \\
\hline C & 0.363792 & -4.816238 & 2.743576 & $\mathrm{H}$ & -3.861474 & -2.910601 & -1.620685 \\
\hline $\mathrm{C}$ & -2.936379 & -4.035500 & -0.007096 & $\mathrm{H}$ & -4.998915 & -3.489936 & -0.400320 \\
\hline C & -3.781590 & 0.427633 & -4.073848 & $\mathrm{H}$ & 1.395330 & 4.046058 & -4.871050 \\
\hline C & -0.236458 & 2.969842 & -3.919040 & $\mathrm{H}$ & 1.803793 & 3.373517 & -3.292675 \\
\hline $\mathrm{C}$ & -2.857058 & -3.350992 & 1.230104 & $\mathrm{H}$ & 0.780633 & 4.815188 & -3.382239 \\
\hline $\mathrm{C}$ & -1.286011 & 3.144192 & -2.983587 & $\mathrm{H}$ & 1.228077 & -4.148195 & 2.611828 \\
\hline C & 3.978018 & 0.587048 & 2.390030 & $\mathrm{H}$ & 0.723660 & -5.841888 & 2.599109 \\
\hline $\mathrm{C}$ & -1.776880 & -3.582377 & 2.110028 & $\mathrm{H}$ & 0.016855 & -4.691411 & 3.776006 \\
\hline C & -2.418266 & 2.300792 & -3.016718 & $\mathrm{H}$ & -3.744459 & -0.443549 & -3.402801 \\
\hline $\mathrm{C}$ & 4.022695 & 1.698201 & 1.519249 & $\mathrm{H}$ & -3.924489 & 0.049345 & -5.092662 \\
\hline $\mathrm{C}$ & 1.891737 & -0.137256 & 3.491654 & $\mathrm{H}$ & -4.656419 & 1.019136 & -3.777558 \\
\hline C & -1.601657 & -2.744403 & 3.337652 & $\mathrm{H}$ & 1.708324 & -0.673736 & 2.565915 \\
\hline $\mathrm{C}$ & -3.703413 & -1.091642 & 0.713145 & $\mathrm{H}$ & -2.899671 & -1.069362 & -0.015702 \\
\hline C & -3.449948 & 2.355620 & -1.932991 & $\mathrm{H}$ & 0.420017 & 2.833797 & -1.043484 \\
\hline C & -0.111263 & 3.770404 & -0.906187 & $\mathrm{H}$ & 0.140753 & -1.861217 & 2.505952 \\
\hline $\mathrm{C}$ & 3.201110 & 2.917151 & 1.800759 & $\mathrm{H}$ & -2.797547 & 0.473968 & -1.204397 \\
\hline C & 3.065657 & 0.604425 & 3.578178 & $\mathrm{H}$ & 1.933625 & 2.466136 & 0.159208 \\
\hline C & -0.510050 & -1.880688 & 3.374312 & $\mathrm{H}$ & -4.996063 & -3.199339 & 3.040781 \\
\hline $\mathrm{C}$ & -3.824542 & -2.237316 & 1.492450 & $\mathrm{H}$ & -2.532462 & 5.595313 & -2.438953 \\
\hline C & -3.522406 & 1.269627 & -1.065492 & $\mathrm{H}$ & 4.265603 & 1.869613 & 4.863550 \\
\hline C & -1.084797 & 4.096174 & -1.845409 & $\mathrm{H}$ & -3.304810 & -3.481396 & 4.457223 \\
\hline C & 2.122381 & 3.174419 & 0.959826 & $\mathrm{H}$ & -4.323697 & 4.280297 & -2.414458 \\
\hline C & -0.214235 & -1.073349 & 4.493933 & $\mathrm{H}$ & 4.329322 & 3.651701 & 3.498732 \\
\hline $\mathrm{C}$ & -4.868811 & -2.299869 & 2.443216 & $\mathrm{H}$ & -2.878592 & -2.040760 & 6.436882 \\
\hline C & -4.501825 & 1.160569 & -0.055065 & $\mathrm{H}$ & -6.012225 & 4.192302 & -0.614726 \\
\hline C & -1.787522 & 5.315078 & -1.698025 & $\mathrm{H}$ & 2.900594 & 5.658744 & 3.829319 \\
\hline $\mathrm{C}$ & 1.305202 & 4.317611 & 1.096476 & $\mathrm{H}$ & 2.638543 & 1.830143 & 6.745366 \\
\hline C & 3.339263 & 1.307148 & 4.774733 & $\mathrm{H}$ & -6.535353 & -1.279418 & 3.343754 \\
\hline C & 0.966472 & -0.224056 & 4.554481 & $\mathrm{H}$ & -2.069496 & 7.088360 & -0.505337 \\
\hline C & -2.460479 & -2.797124 & 4.461879 & $\mathrm{H}$ & 4.832786 & 3.760189 & -0.001532 \\
\hline C & -4.588008 & 0.003277 & 0.823145 & $\mathrm{H}$ & 3.984945 & 2.922514 & -1.303121 \\
\hline C & -4.369597 & 3.415666 & -1.757447 & $\mathrm{H}$ & 5.746592 & 2.802038 & -1.206930 \\
\hline C & 0.216859 & 4.615224 & 0.176208 & $\mathrm{H}$ & -9.120063 & 2.454951 & 0.342256 \\
\hline C & 3.479078 & 3.830439 & 2.845410 & $\mathrm{H}$ & -7.959362 & 3.228643 & -0.785878 \\
\hline $\mathrm{C}$ & 1.240648 & 0.521914 & 5.747023 & $\mathrm{H}$ & -8.900111 & 4.240834 & 0.364601 \\
\hline C & -2.220656 & -1.999734 & 5.572632 & $\mathrm{H}$ & -9.201136 & 0.828561 & 1.806026 \\
\hline C & -5.622463 & -0.061459 & 1.809503 & $\mathrm{H}$ & -9.002224 & 2.329241 & 2.776754 \\
\hline $\mathrm{C}$ & -5.335682 & 3.355157 & -0.761118 & $\mathrm{H}$ & -9.399642 & 0.771235 & 3.589455 \\
\hline C & -0.524291 & 5.832426 & 0.328586 & $\mathrm{H}$ & -0.106627 & 6.279180 & 4.825376 \\
\hline C & 2.687105 & 4.955211 & 3.028673 & $\mathrm{H}$ & -0.892762 & 7.671694 & 3.996282 \\
\hline C & 2.439037 & 1.277519 & 5.830931 & $\mathrm{H}$ & 0.420427 & 7.958986 & 5.198281 \\
\hline C & -1.101137 & -1.129329 & 5.617393 & $\mathrm{H}$ & 1.758590 & -0.142508 & 8.988116 \\
\hline C & -5.738239 & -1.230146 & 2.607536 & $\mathrm{H}$ & 0.024216 & -0.107378 & 9.469459 \\
\hline C & -5.451587 & 2.221813 & 0.087877 & $\mathrm{H}$ & 1.117804 & 1.249137 & 9.933141 \\
\hline C & -1.521916 & 6.157435 & -0.626899 & $\mathrm{H}$ & 0.206188 & 9.119781 & 0.399691 \\
\hline $\mathrm{C}$ & 1.596346 & 5.228483 & 2.163484 & $\mathrm{H}$ & 0.480847 & 9.229179 & 2.175001 \\
\hline
\end{tabular}




\begin{tabular}{llllllll}
\hline $\mathbf{C}$ & -0.828323 & -0.314556 & 6.775861 & $\mathrm{H}$ & -1.028710 & 9.899623 & 1.451548 \\
$\mathbf{C}$ & -6.486731 & 2.127371 & 1.093705 & $\mathrm{H}$ & -3.105719 & 1.031561 & 7.108837 \\
$\mathbf{C}$ & 0.784504 & 6.410413 & 2.326306 & $\mathrm{H}$ & -1.839864 & 1.693672 & 8.208984 \\
$\mathbf{C}$ & 0.306477 & 0.467825 & 6.845821 & $\mathrm{H}$ & -3.161098 & 0.659126 & 8.868556 \\
\hline
\end{tabular}

\section{References.}

(S1) Han, Q.; Li, Q. J.; He, J. M.; Hu, B. J.; Tan, H. W.; Abliz, Z.; Wang, C. H.; Yu, Y. H.; Yang, H. B. J. Org. Chem. 2011, 76, 9660-9669.

(S2) Boden, B. N.; Hui, J. K. H.; MacLachlan, M. J. J. Org. Chem. 2008, 73, 8069-8072.

(S3) He, Z. K.; Xu, X. M.; Zheng, X.; Ming, T.; Miao, Q. Chem. Sci. 2013, 4, 4525-4531.

(S4) Amato, F.; Phulwale, B. V.; Mazal, C.; Havel, J. Rapid Commun. Mass Spectrom. 2015, 29, 1125-1134.

(S5) Venkataramana, G.; Dongare, P.; Dawe, L. N.; Thompson, D. W.; Zhao, Y.; Bodwell, G. J. Org. Lett. 2011, 13, 2240-2243.

(S6) Yang, J. S.; Huang, H. H.; Lin, S. H. J. Org. Chem. 2009, 74, 3974-3977.

(S7) Delley, B. J. Chem. Phys. 1990, 92, 508-517.

(S8) Delley, B. J. Chem. Phys. 2000, 113, 7756-7764.

(S9) Monkhorst, H. J.; Pack, J. D. Phys. Rev. B 1976, 13, 5188-5192.

(S10) Perdew, J. P.; Burke, K.; Ernzerhof, M. Phys. Rev. Lett. 1996, 77, 3865-3868.

(S11) Grimme, S. J. Comput. Chem. 2006, 27, 1787-1799.

(S12) Segawa, Y.; Omachi, H.; Itami, K. Org. Lett. 2010, 12, 2262-2265.

(S13) Black, C. B.; Andrioletti, B.; Try, A. C.; Ruiperez, C.; Sessler, J. L. J. Am. Chem. Soc. 1999, 121, 10438-10439.

(S14) Isobe, H.; Hitosugi, S.; Yamasaki, T.; Iizuka, R. Chem. Sci. 2013, 4, 1293-1297.

(S15) Lu, D. P.; Zhuang, G. L.; Wu, H. T.; Wang, S.; Yang, S. F.; Du, P. W. Angew. Chem. Int. Ed. 2017, 56, 158-162. 